\title{
High-energy neutrino flux from individual blazar flares
}

\author{
Foteini Oikonomou* \\ European Southern Observatory, Garching bei München, Germany \\ E-mail: foikonom@eso.org
}

Kohta Murase

The Pennsylvania State University, University Park, USA

Kyoto University, Kyoto, Japan

E-mail: murase@psu.edu

\section{Paolo Padovani}

European Southern Observatory, Garching bei München, Germany

Associated to INAF - Osservatorio Astronomico di Roma, Monteporzio Catone, Italy

E-mail: ppadovandeso.org

\section{Elisa Resconi}

Technische Universität München, Garching bei München, Germany

E-mail: elisa.resconi@tum.de

\section{Peter Mészáros}

The Pennsylvania State University, University Park USA

E-mail: nnp@psu.edu; peter.meszaros7@gmail.com

Motivated by the recently reported evidence of an association between a high-energy neutrino and a $\gamma$-ray flare from the blazar TXS 0506+056, we calculate the expected high-energy neutrino signal from past, individual flares, from twelve blazars, selected in declinations favourable for detection with IceCube. To keep the number of free parameters to a minimum, we mainly focus on BL Lac objects and assume the synchrotron self-Compton mechanism produces the bulk of the high-energy emission. We consider a broad range of the allowed parameter space for the efficiency of proton acceleration, the proton content of BL Lac jets, and the presence of external photon fields. To model the expected neutrino fluence we use simultaneous multi-wavelength observations. We find that in the absence of external photon fields and with jet proton luminosity normalised to match the observed production rate of ultra-high-energy cosmic rays, individual flaring sources produce a modest neutrino flux in IceCube, $N_{v_{\mu},>100 \mathrm{TeV}}^{\mathrm{IC}, 10 \mathrm{yr}} \lesssim 10^{-3}$ muon neutrinos with energy exceeding $100 \mathrm{TeV}$, stacking ten years of flare periods selected in the $>800 \mathrm{MeV}$ Fermi energy range, from each source. Under optimistic assumptions about the jet proton luminosity and in the presence of external photon fields, we find that the two most powerful sources in our sample, AO 0235+164, and OJ 287, would produce, in total, $N_{v_{\mu}, \text { all flares, }>100 \mathrm{TeV}}^{\mathrm{IC} \times 10,10 \mathrm{yr}} 3$ muon neutrinos during Fermi flaring periods, in future neutrino detectors with total instrumented volume $\sim$ ten times larger than IceCube, or otherwise, constrain the proton luminosity of blazar jets.

36th International Cosmic Ray Conference -ICRC2019-

July 24th - August 1st, 2019

Madison, WI, U.S.A. 


\begin{tabular}{ccccccc}
\hline Source & $\mathrm{z}$ & decl. & UT & $\Delta T$ & Data Ref. & Selection Band \\
\hline 3C 66A & 0.34 & +43.04 & Oct 2008 & 14 & 1 & VERITAS \\
AO 0235+164 & 0.94 & +16.62 & Oct 2008 & 84 & 2 & Fermi \\
Mrk 421 & 0.031 & +38.21 & Mar 2010 & 13 & 3 & MAGIC \\
PG 1553+113 & $\sim 0.4$ & +11.19 & Apr 2012 & 30 & 4,5 & MAGIC \\
1ES 1959+650a & 0.048 & +20.00 & May 2012 & 46 & 6 & VERITAS \\
Mrk 501 & 0.033 & +39.76 & Jun 2012 & 21 & 7 & MAGIC \\
S5 0716+714 & $<0.32$ & +71.37 & Jan 2015 & 14 & 8,9 & Optical/NIR \\
S4 0954+65 & $\geq 0.45$ & +65.57 & Feb 2015 & 28 & 10,11 & Fermi \\
BL Lac & 0.07 & +42.28 & Jun 2015 & 7 & 12 & Fermi \\
S2 0109+22 & 0.36 & +22.74 & Jul 2015 & 21 & 13 & Fermi \\
1ES 1959+650b & 0.048 & +20.00 & Sep 2015 & 84 & 14 & Fermi \\
OJ 287 & 0.306 & +20.11 & Dec 2015 & 7 & 15 & theoretical \\
TXS 0506+056 & 0.3365 & +5.70 & Sep 2017 & 175 & 16 & Fermi \\
\hline
\end{tabular}

Table 1: The list of flares studied in this work. For each flare the table lists the redshift (or assumed redshift) of the source, declination, the month of onset of the flare, the flare duration, which we determined by analysis of the $>800 \mathrm{MeV}$ FAVA [8] lightcurves ${ }^{\star}$, references where the flare campaigns were presented, and selection band for each flare. The references are: 1 - [9], 2 - [10], 3 - [11], 4,5 - [12, 13]. 6 - [14], 7 - [15], 8 - [16], 9-[17], 10, 11 - [18, 19], 12 - [20], 13 - [21], 14 - [22], 15 - [23], 16 - [1].

* Note that for the flares of Mrk 421, PG 1553+113, and 1ES 1959+650a for which no flaring activity was detectable in the FAVA data we used the flare duration as determined from the MAGIC, Swift (XRT), and optical data respectively.

\section{Introduction}

The IceCube Collaboration has recently reported the observation of a $\gtrsim 290 \mathrm{TeV}$ muon neutrino, IceCube-170922A, coincident with a $\sim 6$-month-long $\gamma$-ray flare of the blazar TXS 0506+056 [1] at redshift $z=0.3365$ [2]. The neutrino detection prompted electromagnetic follow-up of the event, and the blazar flare was detected by several instruments, including MAGIC at energies exceeding $>100 \mathrm{GeV}$. The correlation of the neutrino with the flare of TXS 0506+056 is inconsistent with arising by chance at the $3 \sigma$ level. The reported association of IceCube-170922A with, and flaring activity by, TXS $0506+056[1,3,4,5,6]$ prompt us to investigate the number of expected neutrino events from earlier blazar flares, that were also detectable by IceCube. A full exposition of the analysis is given in [7]. Here we present the flare data used (Sec. 2), key methods (Sec. 3) and results of the study (Sec. 4).

\section{Dataset}

We collected publicly available data for recorded flaring episodes of 12 Fermi bright BL Lacs, for which simultaneous (or semi-simultaneous) observations exist, with sufficient spectral coverage as to infer the main characteristics of the SED during the flare with reasonable confidence (for example the peak synchrotron frequency and peak synchrotron flux). Neutrino production is expected to be more efficient in FSRQs than in BL Lac objects due to the higher-powers and existence of

\footnotetext{
* Speaker.
} 
external photon fields [24]. However, these also introduce additional free parameters. Therefore, in this work we have focused on objects classified as BL Lacs, in which the bulk of the emission can in general be attributed to synchrotron and synchrotron self-Compton (SSC) processes. The details of all the flares in our sample are given in Tab. 1.

\section{Methods}

We consider the interactions of protons in the jet emitting region (blob), with photons comoving with the relativistic blob. The neutrino spectrum produced by pion and muon decay following $p \gamma$ interactions is calculated as, $\varepsilon_{v}^{\prime 2} \mathrm{~d} N_{v_{\mu}}^{\prime} / \mathrm{d} \varepsilon_{v_{\mu}}^{\prime} \approx 3 / 8 \cdot f_{p \gamma}^{\prime} \cdot \varepsilon_{p}^{\prime 2} \mathrm{~d} N_{p}^{\prime} \mathrm{d} \varepsilon_{p}^{\prime}$, where $\mathrm{d} N_{p}^{\prime} / \mathrm{d} \varepsilon_{p}^{\prime}$ is the number of protons of energy $\varepsilon_{p}^{\prime}$. We consider all the cooling processes that affect protons and secondary mesons in the jet, which determine $f_{p \gamma}^{\prime}$, the efficiency of neutrino production in each source. Our approach is semianalytic and has the advantage of fast computation with reasonable accuracy, which allows for parameter-space scans and application to multiple sources. We have benchmarked our method against the time-dependent radiation modelling of [5] and find agreement within a factor of two for the expected neutrino flux between the two approaches.

For the magnetic field strength, $B$, and doppler factor of the motion, $\delta$, we assume the values derived from detailed modelling of the studied flares in previous literature, as summarised in Tab. 2. For seven of the twelve sources studied a pure SSC model can explain the SED (see Tab. 4 of [7]). For the remaining five, we used parameters derived assuming in addition an external Compton component. Where this is the case, the neutrino prediction in Normalisation A introduced below is conservative as it does not include neutrinos that can potentially be created in interactions with the external photon field.

We normalise the total proton luminosity of the source, $\mathscr{L}_{\mathrm{p}}^{\prime}$, to the total (isotropic equivalent) comoving photon luminosity $\mathscr{L}_{\gamma}^{\prime}$, assuming $\mathscr{L}_{\mathrm{p}}^{\prime}=\xi_{\text {cr }} \mathscr{L}_{\gamma}^{\prime}$, with $\xi_{\text {cr }}$ the baryon loading factor. We assume this relation to hold at all times. The maximum proton energy in each source depends on the balance between the acceleration timescale, $t_{\mathrm{acc}}^{\prime}=\eta \varepsilon_{p}^{\prime} /\left(c e B^{\prime}\right)$, and all other cooling processes. Here, the acceleration efficiency is parametrised by $\eta$, with $\eta=1$ in the Bohm limit, $c$ the speed of light, and $e$ the charge of the electron.

We consider two possible scenarios for the physical parameters in the sources, detailed below:

- Normalisation A (UHECR / conservative): Here, the baryon loading is relatively low, $\xi_{\mathrm{cr}}=10$, and the maximum proton energy is high (the acceleration efficiency is high, with $\eta=1$ ), reaching ultra-high energies. This baryon loading roughly corresponds to the average baryon loading needed for blazars to power the locally observed ultra-high energy cosmic ray (UHECR) production rate, e.g. [25], as calculated in [26]. The neutrino spectra obtained with this model typically peak beyond $10 \mathrm{PeV}$. Neutrinos are produced only in interactions of protons with synchrotron photons in the blob.

- Normalisation B (TXS 0506+056 / optimistic): This is inspired by the most optimistic model of [5], LMPL2b, for the September 2017 flare of TXS 0506+056 (see also their Tab. 7) if IceCube-170922A was indeed produced by TXS 0506+056. The baryon loading factor is high, $\xi_{\text {cr }}=1540$. The maximum proton energy is low (the acceleration efficiency is low 


\begin{tabular}{c|cc|cccccc} 
Source & $\delta$ & $B$ & $\mathscr{L}_{p}^{46}$ & $\mathscr{L}_{E_{\mu}}^{40}$ & $N_{v_{\mu}, \text { flare }}^{\mathrm{IC},>10 \mathrm{TeV}}$ & $N_{v_{\mu}, / \text { year flare }}^{\mathrm{IC},>100 \mathrm{TeV}}$ & $N_{v_{\mu}, \text { all flares }}^{\mathrm{IC},>100 \mathrm{TeV}}$ & $N_{v_{\mu}, 10 \mathrm{yr}}^{\mathrm{IC},>10 \mathrm{TeV}}$ \\
\hline 3C 66A & 40 & 0.02 & 700 & 400 & $1 \mathrm{e}-8$ & $2 \mathrm{e}-7$ & $2 \mathrm{e}-7$ & $1 \mathrm{e}-6$ \\
AO 0235+164 & 20 & 0.22 & $9 \mathrm{e}+3$ & $8 \mathrm{e}+6$ & $5 \mathrm{e}-4$ & $2 \mathrm{e}-3$ & $2 \mathrm{e}-3$ & $3 \mathrm{e}-3$ \\
Mrk 421 & 21 & 0.04 & 10 & 7 & $3 \mathrm{e}-7$ & $7 \mathrm{e}-6$ & $6 \mathrm{e}-5$ & $1 \mathrm{e}-4$ \\
PG 1553+113 & 40 & 0.045 & 700 & $4 \mathrm{e}+3$ & $6 \mathrm{e}-6$ & $5 \mathrm{e}-7$ & $1 \mathrm{e}-5$ & $1 \mathrm{e}-4$ \\
1ES 1959+65a & 25 & 0.01 & 9 & 1 & $2 \mathrm{e}-7$ & $1 \mathrm{e}-6$ & $5 \mathrm{e}-7$ & $8 \mathrm{e}-7$ \\
Mrk 501 & 10 & 0.02 & 7 & 30 & $4 \mathrm{e}-5$ & $7 \mathrm{e}-4$ & $1 \mathrm{e}-3$ & $3 \mathrm{e}-3$ \\
S5 0716+714 & 25 & 0.10 & 400 & $2 \mathrm{e}+4$ & $2 \mathrm{e}-8$ & $4 \mathrm{e}-7$ & $6 \mathrm{e}-7$ & $1 \mathrm{e}-6$ \\
S4 0954+65 & 30 & 0.6 & 600 & $3 \mathrm{e}+4$ & $1 \mathrm{e}-7$ & $1 \mathrm{e}-6$ & $1 \mathrm{e}-7$ & $2 \mathrm{e}-7$ \\
BL Lac & 25 & 0.14 & 50 & 70 & $5 \mathrm{e}-7$ & $2 \mathrm{e}-5$ & $1 \mathrm{e}-5$ & $3 \mathrm{e}-5$ \\
S2 0109+22 & 22 & 0.05 & 200 & $3 \mathrm{e}+3$ & $5 \mathrm{e}-7$ & $1 \mathrm{e}-5$ & $2 \mathrm{e}-5$ & $4 \mathrm{e}-5$ \\
1ES 1959+65b & 25 & 0.01 & 4 & $3 \mathrm{e}-1$ & $1 \mathrm{e}-7$ & $5 \mathrm{e}-7$ & $5 \mathrm{e}-7$ & $8 \mathrm{e}-7$ \\
OJ 287 & 14 & 0.9 & 200 & $2 \mathrm{e}+5$ & $2 \mathrm{e}-5$ & $1 \mathrm{e}-3$ & $9 \mathrm{e}-5$ & $3 \mathrm{e}-4$ \\
TXS 0506+056 & 24 & 0.4 & 300 & $3 \mathrm{e}+4$ & $4 \mathrm{e}-5$ & $7 \mathrm{e}-5$ & $4 \mathrm{e}-5$ & $8 \mathrm{e}-5$ \\
\hline
\end{tabular}

Table 2: The first two columns give the assumed doppler factor, $\delta$, and magnetic field strength, $B$, in Gauss, which are taken from references as listed below ${ }^{\star}$. Further, the assumed lab frame proton luminosity, $\mathscr{L}_{p}^{46}$, in units of $10^{46} \mathrm{erg} \mathrm{s}^{-1}$, the corresponding predicted lab frame muon-neutrino luminosity, $\mathscr{L}_{E_{v_{\mu}}^{40}}$, in units of $10^{40} \mathrm{erg} \mathrm{s}^{-1}$, and the number of predicted muon-neutrino archival events for the flare studied in IceCube, $N_{v_{\mu}}^{\mathrm{IC},>100 \mathrm{fla}}=$ with energy $\geq 100 \mathrm{TeV}$ for the model parameters of Normalisation A are given. In addition, the number of muon neutrinos per year of flaring activity $N_{v_{\mu}, / \text { year flare }}^{\mathrm{IC},>100 \mathrm{TeV}}$ is given. The two rightmost columns give the number of muon neutrinos produced during all the flares identified through the $>800 \mathrm{MeV}$ FAVA tenyear-lightcurve analysis, $N_{v_{\mu}}^{\mathrm{IC},>100 \mathrm{TeV}}$, flares , and the total number of expected neutrinos over the entire ten years of FAVA data available, $N_{v_{\mu}, 10 \mathrm{yr}}^{\mathrm{IC},>100 \mathrm{TeV}}$, in Normalisation A, under the assumption that $N_{v_{\mu}} \propto F_{\mathrm{HE}}^{2}$, with $F_{\mathrm{HE}}$ the flux of the source in the FAVA high-energy bin.

* References for $\delta$ and $B$ : 3C 66A [9], AO 0235+164 [10], Mrk 421 [11], PG 1553+113 [13], 1ES 1959+650 [14], Mrk 501 [15], S5 0716+714 [17], S4 0954+65 [18, 19], BL Lac [20], S2 0109+22 [21], OJ 287 [29], TXS 0506+056 [5].

with $\eta=10^{4}$ ), of order $\mathrm{PeV}$, and as a result the neutrino spectrum typically peaks at $\sim$ sub$\mathrm{PeV}$ energies. Additionally, in Normalisation B, we consider the interactions of protons with an external photon field inspired by the spine-sheath model [27], with spectrum as in the LMPL2b model of [5]. We only consider scenarios where the SSC mechanism is the dominant mechanism responsible for the observed SED, in other words, $u_{\mathrm{syn}}^{\prime}>u_{\mathrm{ext}}^{\prime}$. Generally, the sheath photon field cannot be much higher than the values assumed here, in one-zone models, especially for high-spectral peak BL Lacs [28]. The assumed value of $u_{\mathrm{ext}}^{\prime}$ for each source is given in Tab. 3 .

\section{Results}

Tables 2 and 3 give the expected number of neutrinos in each flare in our sample in the IceCube detector, for Normalisation A and Normalisation B, respectively. We have calculated the expected number of neutrinos by integrating the neutrino spectrum above $100 \mathrm{TeV}$ where the atmospheric background is low. The highest neutrino counts under Normalisation A are expected for $\mathrm{AO} 0235+164$, which is the most luminous source in our sample, despite being the most distant, at redshift $z=0.94$. In the case of Normalisation B, AO $0235+164$ has been modelled with $\xi_{\text {cr }}$ 

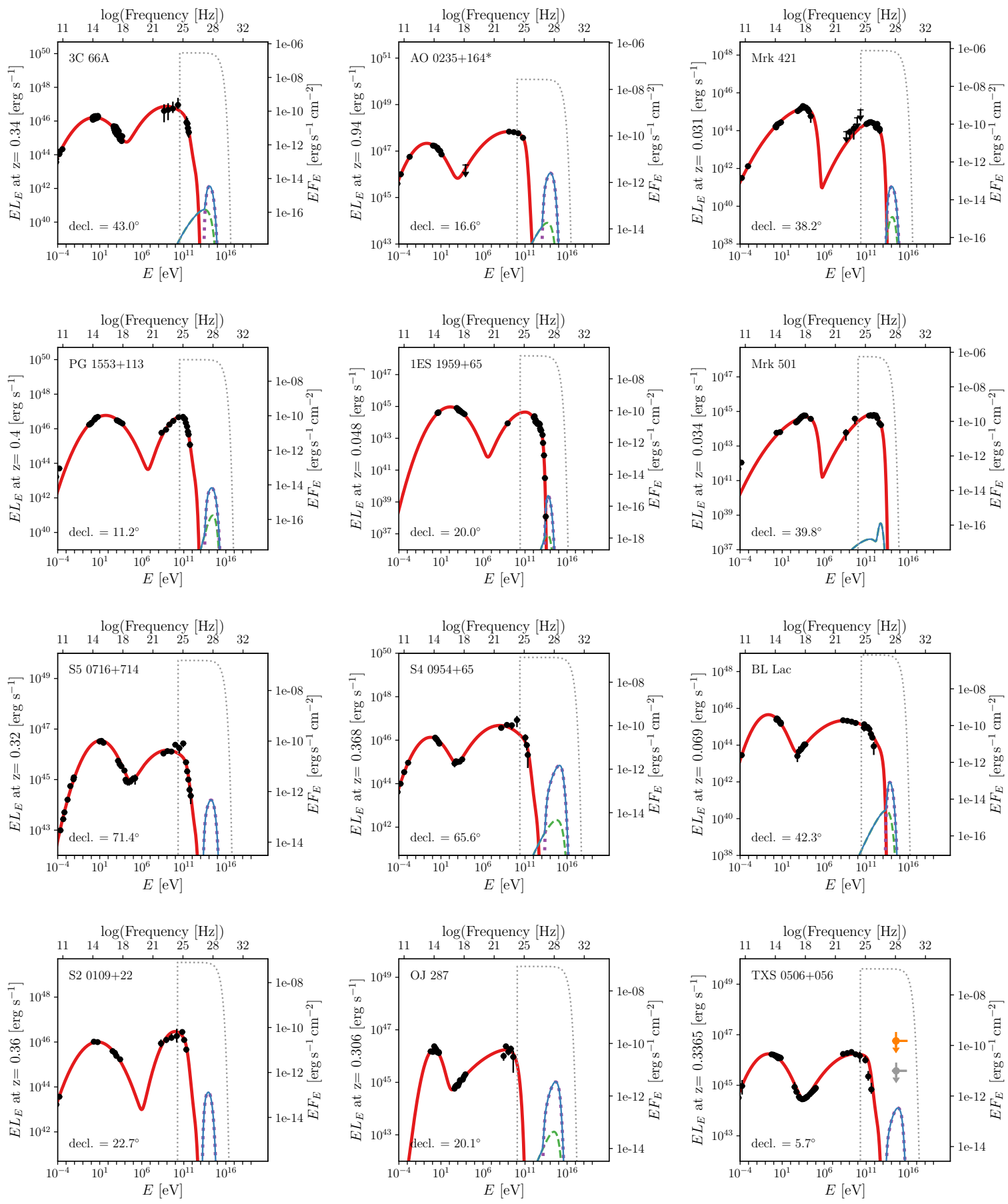

Figure 1: Broadband SED (red), injected proton flux (black dotted lines), and expected instantaneous allflavour neutrino flux (blue) for each for the flares in our sample in Normalisation B in the observer frame. Green dashed lines give the neutrino emission produced in interactions of protons with photons the blob. For some of the SEDs these are very suppressed and not seen in the plots. Purple dotted lines give the neutrino flux produced in interactions of protons with photons of the stationary external field. Blue solid lines give the total neutrino emission. For TXS 0506+056, the data-points give the all-flavour neutrino flux upper limits that would produce on average one detection like IceCube-170922A over a period of 0.5 (orange) and 7.5 years (grey) at the most probable neutrino energy as calculated in [1]. * The star marks that for AO $0235+164$ we have used baryon loading ten times lower than all other sources, $\xi_{\mathrm{cr}}=150$. 


\begin{tabular}{c|c|cccccc} 
Source & $u_{\mathrm{ext}}^{\prime}$ & $\mathscr{L}_{p}^{46}$ & $\mathscr{L}_{v_{\mu}}^{40}$ & $N_{v_{\mu}, \text { flare }}^{\mathrm{IC},>100 \mathrm{TeV}}$ & $N_{v_{\mu}, \text { year flare }}^{\mathrm{IC},>100 \mathrm{TeV}}$ & $N_{v_{\mu}, \text { all flares }}^{\mathrm{IC},>100 \mathrm{TeV}}$ & $N_{v_{\mu}, 10 \mathrm{yr}}^{\mathrm{IC},>100 \mathrm{TeV}}$ \\
\hline 3C 66A & $5 \mathrm{e}-5$ & $1 \mathrm{e}+5$ & 70 & $5 \mathrm{e}-6$ & $1 \mathrm{e}-4$ & $5 \mathrm{e}-5$ & $5 \mathrm{e}-4$ \\
AO 0235+164 & $3 \mathrm{e}-3$ & $1 \mathrm{e}+5^{\star}$ & $8 \mathrm{e}+5^{\star}$ & $3 \mathrm{e}-2^{\star}$ & $1 \mathrm{e}-1^{\star}$ & $1 \mathrm{e}-1^{\star}$ & $2 \mathrm{e}-1^{\star}$ \\
Mrk 421 & $9 \mathrm{e}-5$ & $2 \mathrm{e}+3$ & 5 & $6 \mathrm{e}-5$ & $1 \mathrm{e}-3$ & $1 \mathrm{e}-2$ & $3 \mathrm{e}-2$ \\
PG 1553+113 & $3 \mathrm{e}-3$ & $1 \mathrm{e}+5$ & 200 & $4 \mathrm{e}-5$ & $5 \mathrm{e}-4$ & $7 \mathrm{e}-4$ & $6 \mathrm{e}-3$ \\
1ES 1959+65a & $2 \mathrm{e}-5$ & $1 \mathrm{e}+3$ & $8 \mathrm{e}-2$ & $3 \mathrm{e}-7$ & $2 \mathrm{e}-6$ & $8 \mathrm{e}-7$ & $1 \mathrm{e}-6$ \\
S5 0716+714 & $2 \mathrm{e}-3$ & $6 \mathrm{e}+4$ & $1 \mathrm{e}+4$ & $3 \mathrm{e}-4$ & $8 \mathrm{e}-3$ & $1 \mathrm{e}-2$ & $2 \mathrm{e}-2$ \\
S4 0954+65 & $9 \mathrm{e}-4$ & $9 \mathrm{e}+4$ & $6 \mathrm{e}+4$ & $9 \mathrm{e}-4$ & $1 \mathrm{e}-2$ & $1 \mathrm{e}-3$ & $1 \mathrm{e}-3$ \\
BL Lac & $4 \mathrm{e}-4$ & $8 \mathrm{e}+3$ & 40 & $5 \mathrm{e}-5$ & $2 \mathrm{e}-3$ & $1 \mathrm{e}-3$ & $2 \mathrm{e}-3$ \\
S2 0109+22 & $5 \mathrm{e}-3$ & $4 \mathrm{e}+4$ & $3 \mathrm{e}+3$ & $3 \mathrm{e}-4$ & $6 \mathrm{e}-3$ & $1 \mathrm{e}-2$ & $2 \mathrm{e}-2$ \\
1ES 1959+65b & $1 \mathrm{e}-5$ & 700 & $2 \mathrm{e}-2$ & $2 \mathrm{e}-7$ & $7 \mathrm{e}-7$ & $8 \mathrm{e}-7$ & $1 \mathrm{e}-6$ \\
OJ 287 & $2 \mathrm{e}-3$ & $3 \mathrm{e}+4$ & $8 \mathrm{e}+4$ & $4 \mathrm{e}-2$ & 2 & $2 \mathrm{e}-1$ & $7 \mathrm{e}-1$ \\
TXS 0506+56 & $7 \mathrm{e}-4$ & $5 \mathrm{e}+4$ & $2 \mathrm{e}+4$ & $2 \mathrm{e}-2$ & $4 \mathrm{e}-2$ & $2 \mathrm{e}-2$ & $4 \mathrm{e}-2$ \\
\hline
\end{tabular}

Table 3: The first column gives the assumed energy density of the external radiation field in Normalisation $\mathrm{B}$ in the comoving frame, $u_{\text {ext }}^{\prime}$, in units of erg $\mathrm{cm}^{-3}$. Columns $2-7$ are the same as columns $3-8$ of Tab. 2 but for Normalisation B, which includes the expected number of neutrinos in interactions with an external photon field. ${ }^{\star}$ Star superscripts denote that for AO $0235+164$ we have used baryon loading factor ten times lower than all other sources $\xi_{\text {cr }}=150$. Mrk 501 is not shown here as the expected number of neutrinos in this model is consistent with zero.

ten times lower than all other sources, as otherwise the secondary electromagnetic emission from proton interactions inside the source would exceed the observed SED of the source. The highest neutrino counts are expected in this case from AO 0235+164 and from the flares of OJ 287 and TXS 0506+056 which are some of the brightest sources in our sample.

The predicted neutrino event counts for Normalisation A are in general modest. The expected neutrino counts increase by up to three orders of magnitude for Normalisation B (Tab. 3) due to the much higher baryon loading and neutrino production in interactions of protons with an external photon field. With Normalisation A, the flare-only neutrino signal is too low to be constrained even with future detectors such as the proposed IceCube Gen2.

Fig. 1 shows the expected neutrino flux from each flare in our sample assuming Normalisation B. For the two most powerful neutrino emitters, AO 0235+164 and OJ 287, a future neutrino network with effective volume ten times larger than IceCube in IC86 configuration, (denoted IC $\times 10$ ) with the combined exposure of the proposed IceCube Gen2, GVD, KM3NeT, and ONC [30, 31, 32, 33], would detect $N_{v_{\mu}, \text { all flares, }>100 \mathrm{TeV}}^{\mathrm{IC} \times 10,10 \mathrm{yr}} \approx 3$. In the absence of the muon-neutrino signal from these two sources, assuming a flare pattern identical to that in the past decade, the baryon loading will be constrained to $\xi_{\text {cr }} \lesssim 1250$ if the energy density of the external photon target field can be probed. For a larger number of such powerful sources the constraint on $\xi_{\text {cr }}$ scales down linearly with the number of expected neutrinos. To gauge the possible reach of future instruments we considered the 34 BL Lac objects extracted from the 1-Jy catalogue [34, 35], and found that using model assumptions equivalent to those described thus far, the future neutrino facilities will be able to constrain $\xi_{\text {cr }} \lesssim 170$.

We have undertaken a study of systematic uncertainties arising from absence of precise knowledge of the physical conditions in the source and their effect on the expected neutrino flux. The latter is particularly sensitive to $\delta$, the presence/absence and energy density of external photon 

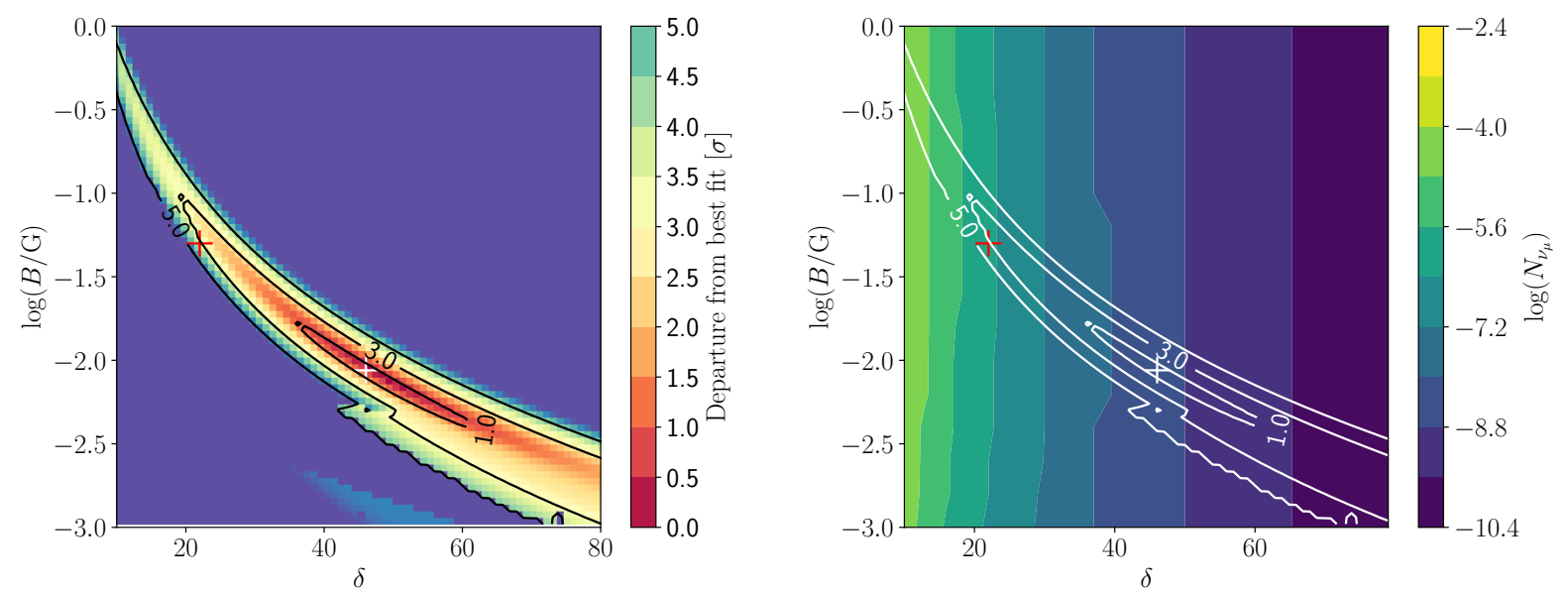

Figure 2: Left: Systematic uncertainty on the determination of $\delta$ and $B$ (in Gauss) during the 21-day, July 2015, flare of S2 0109+22. The colormap gives the departure from the best fit in units of $\sigma$ (standard deviations), i.e. realisations with departure $\leq 2 \sigma$ encompass the $95 \%$ allowed region assuming a Gaussian distribution. Right: Number of muon neutrinos expected to be seen in IceCube (IC86) for each combination of $\delta$ and $B$. The red crosses (in both panels) mark the values of $\delta$ and $B$ assumed in the present study. The white crosses mark the best-fit values of $\delta$ and $B$ as determined with the log-parabolic SSC model fit.

fields, $\xi_{\mathrm{CR}}$, and $B$. Here we examine systematic uncertainties that arise from the uncertainty in $\delta$, and $B$, in the simplest scenario in which external photon fields are absent, for a fixed value of $\xi_{\mathrm{cr}}$. We use the July 2015 flare of S2 0109+22, assuming Normalisation A, for illustration. The left panel of Fig. 2 illustrates the parameter space allowed for $\delta$ and $B$ from the SED of S2 0109+22 during its 2015 flare. It was obtained by scanning over $\delta$ and $B$, performing at each step a double $\log$-parabolic fit which corresponds to the tested values of $\delta$ and $B$ in the SSC model (see Eq. 1, 2 of [7]), and calculating the $\chi^{2}$ of every fit. The right panel of Fig. 2 gives the expected number of muon neutrinos from the flare of S2 0109+22, with Normalisation A, while scanning over $\delta$ and $B$, integrated over neutrino energies above $100 \mathrm{TeV}$. Within the $1 \sigma$ error-region the neutrino expectation varies by more than two orders of magnitude. The literature values of $\delta$ and $B$ fall $3 \sigma$ away from the best-fit log-parabolic parameters. The expected number of neutrinos in the two models varies by $\sim 1.5$ orders of magnitude. One would expect an increase in the expected neutrino counts with increasing $B$, due to the increased maximum proton energy that can be reached in the source in the latter case. However, as the IceCube exposure decreases fast beyond $\sim 10^{16} \mathrm{eV}$ at the declination of $\mathrm{S} 20109+22$, there is no significant increase in the neutino counts expected in this example (see Fig. 2 of [7]). Additional constraints, beyond the shape of the SED in the SSC scenario investigated here can be imposed to exclude certain regions of the parameter space; for example, rapid variability may impose a limit on $\delta$ on a source-by-source basis.

Future, precise, astronomical observations will help narrow-down the allowed parameter range for $\delta$ and $B$, and external photon fields. It will then be possible to constrain or determine $\xi_{\text {cr }}$ with neutrino telescopes, with the method outlined above. 


\section{References}

[1] IceCube Collaboration, Fermi-LAT et al., Science 361 (2018) eaat1378 [1807. 08816].

[2] S. Paiano, R. Falomo et al., ApJ 854 (2018) L32 [1802 . 01939].

[3] MAGIC collaboration, ApJ 863 (2018) L10 [1807. 04300].

[4] VERITAS collaboration, Astrophys. J. 861 (2018) L20 [1807.04607].

[5] A. Keivani, K. Murase et al., ApJ 864 (2018) 84 [1807. 04537 ].

[6] P. Padovani, P. Giommi et al., MNRAS 480 (2018) 192 [1807. 04461 ].

[7] F. Oikonomou, K. Murase et al., to appear in MNRAS (2019) [1906.05302].

[8] Fermi-LAT Collaboration, ApJ 846 (2017) 34 [1612 . 03165].

[9] Fermi-LAT Collaboration,, VERITAS Collaboration, et al., ApJ 726 (2011) 43 [1011. 1053].

[10] Fermi-LAT Collaboration,, R. Moderski et al., ApJ 751 (2012) 159 [1207. 2932].

[11] MAGIC, VERITAS collaboration, A\&A 578 (2015) A22 [1412 . 3576].

[12] H.E.S.S. collaboration, ApJ 802 (2015) 65 [1501. 05087].

[13] MAGIC Collaboration, F. D’Ammando et al., MNRAS 450 (2015) 4399 [1 408 . 1975].

[14] VERITAS Collaboration,, M. Böttcher et al., ApJ 797 (2014) 89.

[15] MAGIC Collaboration,, FACT Collaboration, et al., A\&A 620 (2018) A181 [1808 . 0430 0].

[16] S. Chandra, H. Zhang et al., ApJ 809 (2015) 130 [1507. 06473].

[17] M. Manganaro, G. Pedaletti et al., Galaxies 4 (2016) 69 [170 4 . 03238].

[18] Y. T. Tanaka, J. Becerra Gonzalez et al., PASJ 68 (2016) 51 [1604 . 05427].

[19] MAGIC Collaboration et al., A\&A 617 (2018) A30 [1801.04138].

[20] MAGIC Collaboration, F. D’Ammando et al., A\&A 623 (2019) A175 [1901. 01733].

[21] MAGIC Collaboration, Fermi-LAT Collaboration et al., MNRAS 480 (2018) 879 [1807. 020 95].

[22] N. Kaur, S. Chandra et al., ApJ 846 (2017) 158.

[23] P. Kushwaha, A. C. Gupta et al., MNRAS 473 (2018) 1145 [1709. 04957 ].

[24] A. M. Atoyan and C. D. Dermer, ApJ 586 (2003) 79 [astro-ph / 0209231 ].

[25] E. Waxman, ApJ 452 (1995) L1 [astro-ph/ 9508037 ].

[26] K. Murase, Y. Inoue et al., Phys. Rev. D 90 (2014) 023007 [1 403.4089 ].

[27] G. Ghisellini, F. Tavecchio et al., A\&A 432 (2005) 401 [astro-ph/ 0406093 ].

[28] F. Tavecchio, F. Oikonomou et al., MNRAS, in press (2019) [1906.02521].

[29] P. Kushwaha, A. C. Gupta et al., MNRAS 479 (2018) 1672 [1803.10213].

[30] ICECube-Gen2 Collaboration collaboration, IceCube-Gen2: A Vision for the Future of Neutrino Astronomy in Antarctica, 2014, 1412.5106.

[31] KM3NET collaboration, KM3NeT Collaboration, KM3NeT: Technical Design Report. 2009.

[32] Baikal-GVD Collaboration, Baikal-GVD: status and prospects, Aug, 2018, 1808 . 10353.

[33] STRAW collaboration, JINST 14 (2019) P02013 [1810 . 13265].

[34] M. Stickel, P. Padovani et al., ApJ 374 (1991) 431.

[35] H. Kuehr, A. Witzel et al., A\&AS 45 (1981) 367. 